\title{
Les Annales françaises de médecine d'urgence : première année, premier bilan
}

\author{
Annales françaises de médecine d'urgence: first year, first balance sheet
}

\author{
B. Riou \\ (C) SFMU et Springer-Verlag France 2011
}

Voici un an, la Société française de médecine d'urgence (SFMU) créait, en partenariat avec Springer, les Annales françaises de médecine d'urgence (AFMU), sa nouvelle revue d'expression scientifique. Il nous incombe d'établir un bilan de cette première année de fonctionnement visà-vis de notre mandant, la SFMU, des auteurs qui ont accepté de nous confier leurs manuscrits, et de nos lecteurs, en particulier les membres de la SFMU.

L'objectif de la SFMU était de faire de cette revue la première des revues francophones de médecine d'urgence. Le contrat est rempli si l'on considère le nombre de personnes qui reçoivent les AFMU, près de 2000 cette année, la régularité de parution tout comme la diversité et la qualité des articles publiés. Le Syndicat de la presse et de l'édition des professions de santé (SPEPS) a d'ailleurs décerné son premier prix, dans la catégorie « travaux originaux », à un article paru dans les AFMU et intitulé Préparation des services d'accueil des urgences (SAU) français à la prise en charge des urgences vitales de l'enfant [1]. À nos lecteurs de dire si les AFMU ont répondu à leur attente à travers l'enquête de satisfaction qui sera lancée en 2012 auprès de notre lectorat.

L'anglais a depuis longtemps gagné la bataille de la recherche scientifique, et il faut espérer qu'un nombre de plus en plus grand d'urgentistes français publie les meilleurs travaux de recherche dans des revues scientifiques d'excellence, donc de langue anglaise. Néanmoins, une revue francophone est indispensable pour publier des articles de formation continue d'excellent niveau dans ce domaine si varié de la médecine d'urgence et qui évolue si vite. Une revue francophone est également indispensable pour publier des articles originaux de qualité qui ne trouvent pas leur place dans les revues de langue anglaise, notamment du fait des spécificités nationales d'organisation de la médecine

\section{B. Riou $(\bowtie)$}

Service d'accueil des urgences, CHU Pitié-Salpêtrière,

AP-HP, université Pierre-et-Marie-Curie-Paris-VI,

47-83, boulevard de l'Hôpital, F-75651 Paris cedex 13, France

e-mail : bruno.riou@psl.aphp.fr d'urgence. Le travail de Claudet et Grouteau [1] est l'exemple même de ces travaux de qualité. Au cours de l'année 2011, nous avons également publié la traduction française d'articles d'équipes françaises parus précédemment dans des revues internationales de haut niveau [2]. Ces articles publiés en conformité avec les règles internationales resteront peu nombreux dans les AFMU mais, bien choisis, ils peuvent trouver ici un nouveau lectorat.

Bien qu'étant une revue d'expression scientifique d'une société française - la SFMU - , les AFMU souhaitent se positionner sur le terrain de la francophonie. C'est dans ce sens que nous avons accueilli, au sein du comité de rédaction, Frédéric Thys (Bruxelles, Belgique) et Bertrand Yersin (Lausanne, Suisse) et que les AFMU ont publié des travaux d'équipes de pays francophones $[3,4]$.

Les articles publiés dans les AFMU sont soumis à un processus d'expertise strict et exigeant effectué par des relecteurs extérieurs (peer-review). Ainsi, au cours de cette première année, 176 manuscrits ont été soumis avec un taux d'acceptation global de $65 \%$ (Tableau 1). Si l'on exclut les articles de commande qui ne sont pas soumis à une évaluation par des experts extérieurs (éditoriaux, actualités en médecine d'urgence) ou ceux qui ne peuvent faire l'objet d'aucune modification (recommandations), on arrive à un taux d'acceptation corrigé de $62 \%$. La liste de ces relecteurs a été publiée en fin d'année 2011, et elle montre leur qualité et leur diversité, la plupart ayant une expérience conséquente des publications au niveau international. La qualité d'une revue dépend de la qualité des manuscrits qui lui sont soumis, mais surtout du travail, bénévole, peu reconnu, accompli par ses relecteurs. Je tiens à leur témoigner ici mes remerciements pour le travail accompli au cours de cette première année et je sais pouvoir à nouveau compter sur eux dans l'avenir.

Le processus éditorial est long, passant par les étapes d'expertise, de révisions plus ou moins nombreuses, de mise en production de l'article, de corrections des épreuves, puis finalement de publication, d'abord en ligne puis sur papier. Toutefois, je m'étais engagé auprès de nos auteurs à leur procurer un service rapide. Nous disposons d'un premier 


\begin{tabular}{|c|c|c|}
\hline 2011 & $\begin{array}{l}\text { Nombre } \\
\text { de manuscrits }\end{array}$ & $\begin{array}{l}\text { Acceptation } \\
(\%)\end{array}$ \\
\hline Éditoriaux & 9 & $9(100)$ \\
\hline Articles originaux & 31 & $17(55)$ \\
\hline Mises au point & 31 & $28(90)$ \\
\hline Cas cliniques commentés & 6 & $4(66)$ \\
\hline $\begin{array}{l}\text { Gestes en médecine } \\
\text { d'urgence }\end{array}$ & 6 & $6(100)$ \\
\hline Cas cliniques & 44 & $22(50)$ \\
\hline $\begin{array}{l}\text { Images en médecine } \\
\text { d'urgence }\end{array}$ & 34 & $20(59)$ \\
\hline Recommandations & 6 & $6(100)$ \\
\hline Lettres à la rédaction & 3 & $3(100)$ \\
\hline $\begin{array}{l}\text { Actualités en médecine } \\
\text { d'urgence }\end{array}$ & 6 & $6(100)$ \\
\hline Total & 176 & $114(65)$ \\
\hline
\end{tabular}

chiffre en 2011 qui est le délai moyen pour obtenir une décision sur les manuscrits, délai tellement important pour les auteurs : 25 jours ! Avec « Editorial Manager », le système de soumission en ligne mis à notre disposition par Springer, nous avons un outil qui permet de solliciter rapidement les relecteurs, de leur rappeler automatiquement les délais lorsqu'ils sont dépassés et d'alerter le rédacteur en chef en cas de problème. Ce délai court est à mettre au crédit $\mathrm{du}$ fonctionnement des AFMU et, là encore, les relecteurs doivent être remerciés pour leur célérité à rendre leur expertise alors même qu'il s'agit de personnes souvent très occupées. Par ailleurs, la publication en ligne des articles, dès que les épreuves sont validées et acceptées, permet de raccourcir les délais de publication liés au rythme de parution de la revue papier. Les AFMU ont ainsi rempli leur objectif de réponse rapide donnée aux manuscrits soumis et de publication rapide des manuscrits acceptés.

L'analyse de la réponse initiale apportée aux manuscrits finalement acceptés aux AFMU est intéressante (Fig. 1A). Dans la très grande majorité des cas $(56 \%)$, la réponse initiale indiquait clairement aux auteurs la volonté de publier leur travail, le plus souvent sous réserve des révisions suggérées par les relecteurs et/ou le rédacteur en chef. Cette façon de procéder permet de conforter les auteurs dans le travail de révision, parfois important, qui leur est demandé. Ce positionnement précoce des AFMU n'empêche pas l'exigence de qualité comme en témoignent les révisions multiples parfois demandées (Fig. 1B). Ces révisions successives sont souvent en rapport avec des points de forme, notamment en ce qui concerne les références bibliographiques, gage de la qualité finale des publications.

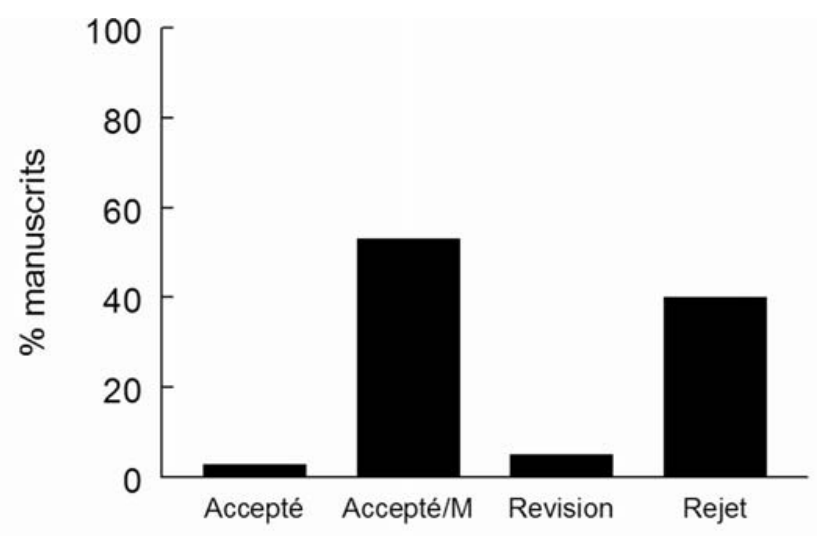

A

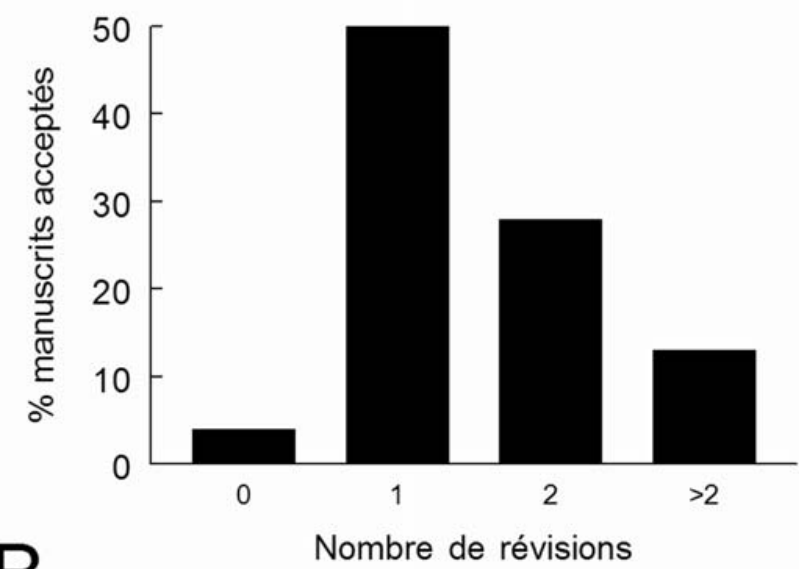

Fig. 1 A. Répartition des décisions initiales (acceptation sans modification, acceptation sous réserve de modifications, révision du manuscrit nécessaire avant décision, rejet) pour les 155 manuscrits de 2011 (hors éditoriaux, actualités en médecine d'urgence et recommandations). B. Nombre de révisions nécessaires pour les 93 manuscrits acceptés

Certaines sections des AFMU ont nécessité un investissement particulier de certains membres du comité de rédaction, et je tenais à les remercier car elles constituent des éléments clés de notre revue. Il s'agit des sections «Actualités en médecine d'urgence » dirigée par Françoise Carpentier (Grenoble), «Images en médecine d'urgence» dirigée par Patrick Ray (Paris) et " Gestes en médecine d'urgence » dirigée par Xavier Combes (Saint-Denis de La Réunion).

Les AFMU s'inscrivent dans les règles et les exigences internationales des revues scientifiques. Ainsi, les instructions aux auteurs des AFMU rappellent leur attachement à ces règles et exigences, notamment en ce qui concerne les dispositions éthiques, légales et réglementaires applicables à la recherche sur des sujets humains et les règles concernant le rôle et la responsabilité scientifique des auteurs. En 2012, les articles acceptés dans les AFMU feront également l'objet 
d'une détection systématique du plagiat afin de participer au mouvement international actuellement en plein essor visant à détecter et à limiter les conséquences de ce comportement illicite $[5,6]$.

Je souhaite à nos lecteurs une excellente année 2012.

\section{Références}

1. Claudet I, Grouteau E (2011) Préparation des services d'accueil des urgences (SAU) français à la prise en charge des urgences vitales de l'enfant. Ann Fr Med Urg 1:5-9
2. André S, Taboulet $P$, Elie $C$, et al (2011) Neutropénie fébrile dans les services d'urgences en France : résultats d'une enquête de pratique multicentrique prospective. Ann Fr Med Urg 1:232-42

3. Slaoui M, Cherradi R, Chapoutot L, Maillier B (2011) Dissection coronaire spontanée compliquée d'infarctus du myocarde : à propos d'un cas. Ann Fr Med Urg 1:138-40

4. De Rook S, Hantson P (2011) Les émulsions lipidiques dans le traitement des effets cardiotoxiques lipophiles autres que les anesthésiques locaux : antidote ou traitement d'exception ? Ann Fr Med Urg 1:26-32

5. Shafer SL (2010) You will be caught! Anesth Analg 112:491-3

6. Bergadaa M (2011) Internet : fraude et déontologie selon les acteurs universitaires. www.responsable.unige.ch (dernier accès $1^{\mathrm{er}}$ novembre 2011) 